\title{
Comparison of a New Magnetic Resonance Imaging Protocol and Magnetic Resonance Follow-Through in Patients with Inflammatory Bowel Disease- A Prospective Pilot Study
}

\author{
Kayalvily Jesuratnam-Nielsen ${ }^{1,2}$, Vibeke B. Løgager ${ }^{1}$, Pia Munkholm ${ }^{2,3}$, Yousef W. Nielsen ${ }^{1}$, \\ Henrik S. Thomsen ${ }^{1,2}$ \\ ${ }^{1}$ Department of Diagnostic Radiology, Copenhagen University Hospital Herlev, Herlev, Denmark \\ ${ }^{2}$ Faculty of Medical and Health Sciences, University of Copenhagen, Copenhagen, Denmark \\ ${ }^{3}$ DEEP-Danish Centre for eHealth \& Epidemiology, North Zealand Hospital, Frederikssund, Denmark \\ Email: Kayalvily.Nielsen@gmail.com
}

Received 10 May 2015; accepted 27 June 2015; published 30 June 2015

Copyright (C 2015 by authors and Scientific Research Publishing Inc.

This work is licensed under the Creative Commons Attribution International License (CC BY).

http://creativecommons.org/licenses/by/4.0/

(c) (i) Open Access

\section{Abstract}

Objective: Inflammatory bowel disease (IBD) is a chronic disease, consisting of two main subgroups: Crohn's disease (CD) and ulcerative colitis (UC). Imaging is an essential component in the treatment of IBD and is used repeatedly to determine activity and severity of inflammatory lesions. The aim of our study was to prospectively obtain pilot data on the accuracy of magnetic resonance imaging with no oral contrast (MRI-NOC) and magnetic resonance follow-through (MRFT) using endoscopy as the reference standard. Materials and Methods: Patients diagnosed with CD and UC referred to magnetic resonance imaging (MRI) were included in this study population. For the evaluation, the bowel was divided into 9 segments. Two radiologists, blinded to clinical findings, evaluated bowel wall thickness, diffusion weighted imaging and mural hyperenhancement. Results: 14 patients ( 9 males and 5 females; median age 41; range 20 - 62) underwent both type of MRI examinations; 9 had CD and 5 had UC. The overall sensitivity for small bowel was zero for both MRI protocols, as neither identified any lesions, whereas in colon the sensitivity ranged from $7 \%$ to $29 \%$ in MRI-NOC and $14 \%$ - 29\% in MRFT. Specificity and accuracy in MRI-NOC ranged from $78 \%$ to $98 \%$ and $74 \%-93 \%$, respectively, in small bowel, and from $90 \%$ to $96 \%$ and $77 \%-82 \%$, respectively, in colon. Specificity and accuracy in MRFT ranged from 83\% to 100\% and 79\% - 95\%, respectively, in small bowel, while it ranged from $93 \%$ to $97 \%$ and $81 \%-85 \%$, respectively, in colon. Conclusion: The location of lesions in the colon combined with the lack of oral contrast in the colon renders MRFT and MRI-NOC functionally identical.

How to cite this paper: Jesuratnam-Nielsen, K., Løgager, V.B., Munkholm, P., Nielsen, Y.W. and Thomsen, H.S. (2015) Comparison of a New Magnetic Resonance Imaging Protocol and Magnetic Resonance Follow-Through in Patients with Inflammatory Bowel Disease-A Prospective Pilot Study. Open Journal of Radiology, 5, 117-124. 


\section{Keywords}

\section{Crohn's Disease, Faecal Calprotectin, Inflammatory Bowel Disease, Magnetic Resonance Imaging, Ulcerative Colitis}

\section{Introduction}

IBD is a common term for two chronic diseases, Crohns disease (CD) and ulcerative colitis (UC). The incidence and prevalence of IBD have increased over the past 20 years in Denmark [1]. The same trend has been observed in Europe and other developed countries [2]. Imaging is an essential component in the treatment of IBD and is used repeatedly to determine activity and severity of inflammatory lesions and chose the right therapy for a given disease severity and extension. Monitoring of $\mathrm{CD}$ and $\mathrm{UC}$ is crucial for the choice of therapeutic strategy in order to personalize medicine and optimize efficacy and compliance [3].

Radiological imaging techniques such as computer tomography, magnetic resonance imaging (MRI), ultrasonography and other imaging modalities such as endoscopy and capsule endoscopy (CE) play a key role in diagnosing and monitoring CD and UC. Despite its diagnostic excellence in the abdomen, computer tomography should be avoided due to the radiation. Ultrasonography has a very good safety profile, but the interpretation of the images is operator dependent. Due to gas in the bowel system, it is sometimes difficult to visualize all parts of the bowel. In CE and endoscopy, it is only possible to evaluate the surface of the bowel wall. CE is well accepted by the patients whereas endoscopy is not favoured by the patients [4]. CE and endoscopy are both contraindicated in patients with suspected stricture.

In recent years, both clinicians and radiologists have become more aware of the potential risk of radiation. It is important to weigh the benefits and drawbacks in every single examination. A single examination that is noninvasive, fast, free of radiation, low cost, patient friendly and excellent in disease diagnostic simply does not exist.

MR follow-through (MRFT) is an emerging technique that meets all the safety criteria. It also has the advantage of enabling evaluation of luminal and transmural extent of inflammation, while showing extra intestinal complications as well. Another emerging diagnostic tool is faecal calprotectin (FC), which is a non-invasive and cheap screening tool to be used as a screening method to monitor IBD relapse and response. However, FC is not a specific protein for IBD because it is also elevated in any other inflammatory condition in the bowel as well as in colorectal cancer. FC is stable up to seven days at room temperature enabling patients to perform the test at home and send the sample by mail to the laboratory. Several studies have shown that elevated FC levels correlate with inflammation in the gastrointestinal tract.

The primary endpoint was to prospectively compare the sensitivity and specificity in MRI with no oral contrast (MRI-NOC), MRFT and FC in patients with known CD and UC using ileo-colonoscopy, sigmoidoscopy and $\mathrm{CE}$ as reference standard for presence and location of inflammation.

\section{Materials and Methods}

\subsection{Study}

This prospective, single-institution study was approved by the Local Committee for Health Research Ethics (No. H-2-2010-149) and the Danish Data Protection Agency (No. 2007-58-0015/HEH.750.8-14). It was registered at Clinicaltrials.gov (No. NCT02255019). Written informed consent was obtained from all patients.

\subsection{Patients}

The study population includes all patients diagnosed with CD and UC referred to MRI. Exclusion criteria were age younger than 18 years, previous moderate or serious reactions to gadolinium-based contrast medium, pregnancy, ferromagnetic implants, estimated glomerular filtration rate $<30 \mathrm{ml} / \mathrm{min} 1.73 \mathrm{~m}^{2}$ or known cancer.

The clinical disease activity in patients with CD was assessed according to the Harvey-Bradshaw index (HBI) and by Simple Clinical Colitis Activity Index (SCCAI) for UC patients. HBI was classified into four categories (normal, mild, moderate and severe) whereas SCCAI was classified into three categories (normal, mild-to- 
moderate and severe).

\subsection{MRI-NOC}

This was the first of the two examination performed. Patients were instructed not to drink or eat for 4 hours prior to the MRI. No bowel cleansing was done. Patients were examined in prone position. All patients were scanned from diaphragm to perineum. Scopolamin butylbromide $20 \mathrm{mg}$ (Buscopan ${ }^{\circledR}$, Boehringer Ingelheim, Ingelheim am Rhein, Germany) was administered twice due to reduce motion artefacts from peristalsis. First time it was given prior to the MRI examination and second time in conjunction with the intravenous contrast agent; 0.1 $\mathrm{mmol} / \mathrm{kg}$ of the gadolinium-based contrast agent (gadoterate-Dotarem ${ }^{\circledR}$, Guerbet, Roissy CDG, France). A maximum of $20 \mathrm{ml}$ gadoterate was injected. All images were transmitted to a "Picture Archiving and Communication System". The average duration of the MRI exam was approximately $30 \mathrm{~min}$.

Both MRI examinations were done in a $1.5 \mathrm{~T}$ scanner (Achieva Philips Healthcare, Best, the Netherlands) equipped with a torso 16-channel phased array coil. The different sequences used in these protocols appear in Table 1.

\subsection{MRFT Protocol}

MRFT was performed two days after the plain MRI. Patients were instructed to drink $1.350 \mathrm{~mL}$ of a barium sulphate suspension (VoLumen ${ }^{\circledR}$; Bracco Diagnostics, Milan, Italy) within 45 - 60 min. This protocol was same as the MRI-NOC.

\subsection{Faecal Calprotectin}

Patients received a faeces sample kit upon admission for the MRI examination. The stool sample should be collected the following day and returned by mail, with the sample containing $5-10 \mathrm{~g}$ of faeces. The stool was analysed by commercial ELISA system (CALPRO AS, Lysaker, Norway) based on antibodies against calprotectin. The calprotectin levels between $0-100 \mu \mathrm{g} / \mathrm{g}$ were considered negative and $>100 \mu \mathrm{g} / \mathrm{g}$ were considered abnormal.

\subsection{Image Analysis}

The bowel from the jejunum to the rectum was evaluated by two radiologists—-with 16 and 2 years MR experience, respectively. The examinations were randomised and the radiologists were blinded to all clinical information. The small bowel consisted of jejunum, ileum and terminal ileum. The colon was divided into six segments:

\section{Table 1. MRI-NOC and MRFT parameters.}

\begin{tabular}{|c|c|c|c|c|c|c|c|}
\hline Parameter & TSE T1 & $\begin{array}{l}\text { SSHSE } \\
\text { fat. sat. }\end{array}$ & SSHSE & DWI & GRE & TSE & $\begin{array}{c}\text { GRE } \\
\text { fat. sat. }\end{array}$ \\
\hline Imaging plans & Coronal & Coronal & Axial & Axial & Axial & Coronal & Coronal \\
\hline $\mathrm{TR} / \mathrm{TE}(\mathrm{ms})$ & $587 / 7$ & $1200 / 80$ & $399 / 80$ & $10813 / 77$ & $3.8 / 1.87$ & $587 / 7$ & $4.4 / 2.1$ \\
\hline Flip angel (degrees) & 90 & 90 & 90 & 90 & 10 & 90 & 90 \\
\hline Field of view (mm) & $400 \times 438$ & $405 \times 405$ & $375 \times 297$ & $300 \times 300$ & $375 \times 295$ & $400 \times 438$ & $450 \times 482$ \\
\hline Selection thickness (mm) & 5 & 7 & 5 & 5 & 3 & 5 & 2 \\
\hline Bandwidth/pixel (Hz) & 218 & 763.1 & 717.6 & 11 & 434.2 & 218 & 378.1 \\
\hline Weighting & $\mathrm{T} 1$ & $\mathrm{~T} 2$ & $\mathrm{~T} 2$ & - & $\mathrm{T} 1$ & $\mathrm{~T} 1$ & $\mathrm{~T} 1$ \\
\hline Matrix & $336 \times 291$ & $252 \times 224$ & $268 \times 182$ & $148 \times 117$ & $188 \times 147$ & $336 \times 291$ & $228 \times 243$ \\
\hline B-value sec/ $/ \mathrm{mm}^{2}$ & & & & $0,50,500,1000$ & & & \\
\hline Intravenous contrast & & & & & + & + & + \\
\hline
\end{tabular}

Abbreviations. DWI: Diffusion Weighted Imaging; GRE: Gradient Echo Sequences; MRFT: Magnetic resonance follow-through; MRI-NOC: Magnetic resonance imaging with no oral contrast; Sat: Saturation; SSHSE: Single-shot Spin Echo; TE: Time to Echo; TR: Time to Repeat; TSE: Turbo Spin Echo. 
caecum, ascending colon, transverse colon, descending colon, sigmoid colon and rectum. For all evaluations, a standardized data sheet was used.

Three and $6 \mathrm{~mm}$ wall thickness were considered normal in the small bowel and colon. Restricted diffusion was defined as a high signal intensity in DWI in 1000 b-value images combined with low signal intensity in Apparent Diffusion Coefficient images. Mural hyperenhancement was considered present when bowel wall had a greater degree of mural enhancement than that of the adjacent loops. Ileo-colonoscopy, sigmoidoscopy and CE served as the reference standard for evaluation of IBD extent and severity.

\subsection{Statistical Analysis}

The sensitivity, specificity and accuracy for MRI-NOC, MRFT and FC were calculated compared to the reference standard; endoscopy and CE. The diagnostic performance of MRI-NOC and MRFT was analysed on a segment-by-segment basis whereas FC was analysed on a per patient basis. Descriptive statistics were applied. Interobserver agreement between paired evaluations of MRI by the two radiologists was assessed through kappa statistics. A kappa value of less than 0.2 was regarded as poor agreement, $0.21-0.4$ as fair agreement, 0.41 0.60 as moderate agreement, $0.61-0.80$ as good agreement and 0.81 or greater as excellent agreement [5]. The statistical analyses were performed using the software package " $\mathrm{R}$ " [6].

\section{Results}

\subsection{Study Population}

15 patients were enrolled in this study from October 2012 to June 2013 and all completed both MR-NOC and MRFT, and all returned the stool sample. However, we had to exclude one patient as no ileo-colonoscopy, sigmoidoscopy or CE was performed. The study group of 14 patients consisted of nine males and five females with a median age of 41 years (range: 20 - 62). Nine had CD and five had UC. The average intake of oral contrast in MRFT was $1163 \mathrm{ml}$. The clinical activity indices HBI for CD patients were normal $(\mathrm{n}=3)$, mild $(\mathrm{n}=3)$, moderate $(n=3)$ with none having severe disease. The SCCAI for UC patients were normal $(n=0)$, mild-to-moderate $(n=2)$ and severe disease $(n=3)$.

\subsection{Magnetic Resonance Imaging}

Surprisingly, the sensitivity for small bowel was zero for both MRI protocols in wall thickening, DWI and hyperenhancement as no lesions were detected (Table 2 and Table 3). However, both protocols missed two lesions in all three categories. The sensitivity, specificity and accuracy in MRI-NOC and MRFT was almost identical for bowel wall thickening, DWI and hyperenhancement in small bowel and in colon. One exception is in the colon where sensitivity, specificity and accuracy for hyperenhancement were significantly better in MRFT compared to MRI-NOC.

\subsection{Faecal Calprotectin}

Sensitivity, specificity and accuracy for FC using endoscopy or CE as the standard of reference were $64 \%$ [CI: 49 - 72], 67\% [CI: 14 - 98] and 64\% [CI: 42 - 78], respectively. The calculations were done on a patient's basis and not on segmental basis.

\subsection{Interobserver Agreement}

The kappa value for interobserver agreement for bowel wall thickening showed moderate agreement $(\kappa=0.43)$, poor agreement for DWI $(\kappa=0.16)$ and fair agreement for hyperenhancement $(\kappa=0.25)$ in MRI-NOC. The MRFT showed fair agreement for wall thickening $(\kappa=0.38)$ and DWI $(\kappa=0.35)$ and good agreement for hyperenhancement $(\kappa=0.65)$.

\section{Discussion}

This pilot study consists of only 14 patients, and the statistical calculations are characterized by the small sample size. Researchers chose only to include patients with known CD or UC to improve the statistics. Endoscopy or 
Table 2. Sensitivity, specificity and accuracy of MRI-NOC in IBD patients.

\begin{tabular}{|c|c|c|c|c|}
\hline & $\begin{array}{c}\text { Sensitivity (\%) } \\
{[95 \% \mathrm{CI}]}\end{array}$ & $\begin{array}{c}\text { Specificity (\%) } \\
{[95 \% \mathrm{CI}]}\end{array}$ & $\begin{array}{c}\text { Accuracy (\%) } \\
{[95 \% \mathrm{CI}]}\end{array}$ & P-value \\
\hline \multicolumn{5}{|l|}{ Wall thickening } \\
\hline Small bowel & $\begin{array}{c}0 \\
{[0-47]}\end{array}$ & $\begin{array}{c}98 \\
{[98-100]}\end{array}$ & $\begin{array}{c}93 \\
{[93-97]}\end{array}$ & 1.000 \\
\hline Colon & $\begin{array}{c}14 \\
{[3-29]}\end{array}$ & $\begin{array}{c}96 \\
{[93-99]}\end{array}$ & $\begin{array}{c}82 \\
{[78-87]}\end{array}$ & 0.192 \\
\hline \multicolumn{5}{|l|}{ DWI } \\
\hline Small bowel & $\begin{array}{c}0 \\
{[0-80]}\end{array}$ & $\begin{array}{c}78 \\
{[78-82]}\end{array}$ & $\begin{array}{c}74 \\
{[74-81]}\end{array}$ & 1.000 \\
\hline Colon & $\begin{array}{c}29 \\
{[10-51]}\end{array}$ & $\begin{array}{c}90 \\
{[86-95]}\end{array}$ & $\begin{array}{c}80 \\
{[74-87]}\end{array}$ & 0.081 \\
\hline \multicolumn{5}{|c|}{ Hyperenhancement } \\
\hline Small bowel & $\begin{array}{c}0 \\
{[0-72]}\end{array}$ & $\begin{array}{c}95 \\
{[95-99]}\end{array}$ & $\begin{array}{c}91 \\
{[91-97]}\end{array}$ & 1.000 \\
\hline Colon & $\begin{array}{c}7 \\
{[0-28]}\end{array}$ & $\begin{array}{c}91 \\
{[90-96]}\end{array}$ & $\begin{array}{c}77 \\
{[75-84]}\end{array}$ & 1.000 \\
\hline
\end{tabular}

Table 3. Sensitivity, specificity and accuracy of MRFT in IBD patients.

\begin{tabular}{ccccc}
\hline & $\begin{array}{c}\text { Sensitivity (\%) } \\
{[95 \% \mathrm{CI}]}\end{array}$ & $\begin{array}{c}\text { Specificity (\%) } \\
{[95 \% \mathrm{CI}]}\end{array}$ & $\begin{array}{c}\text { Accuracy (\%) } \\
{[95 \% \mathrm{CI}]}\end{array}$ & P-value \\
\hline Wall thickening & & & & \\
Small bowel & 0 & 98 & 93 & 1.000 \\
Colon & {$[0-47]$} & {$[98-100]$} & {$[93-97]$} & \\
& 14 & 97 & 83 & 0.128 \\
DWI & {$[3-26]$} & {$[85-100]$} & {$[80-87]$} & \\
Small bowel & & & & \\
& 0 & 83 & 79 & 1.000 \\
Colon & {$[0-79]$} & {$[83-87]$} & {$[79-86]$} & \\
& 21 & 93 & 81 & 0.125 \\
Hyperenhancement & {$[6-41]$} & {$[90-97]$} & {$[76-88]$} & \\
Small bowel & & & & \\
& 0 & 100 & 95 & \\
Colon & {$[0]$} & {$[100]$} & {$[95]$} & 0.012 \\
\hline
\end{tabular}

CE was used to verify the presence of lesions on the MRI. This was done to strengthen the findings in this pilot study.

With endoscopy and CE serving as the reference standard, the sensitivity for inflammation in colon ranged from $14 \%$ to $29 \%$ in both MRI examinations. Whereas, in small bowel the sensitivity was zero in both protocols as they missed to find two lesions. In one CD patient with very mild inflammation in most of the colon, both MRI protocols failed to detect it. It is notable that sensitivity, specificity and accuracy for MRI-NOC and MRFT are very close to each other (Figure 1). However, the small amount of participants is not insignificant.

Jesuratnam-Nielsen et al. performed a study where they compared plain MRI (with no oral or intravenous contrast) with MRFT in 100 IBD patients [7]. They conclude that plain MRI cannot replace MRFT in patients with inflammatory bowel disease. However, they use MRFT as the reference standard. This study was the inspiration to make the current study. Authors therefore altered the plain MRI and gave the patients intravenous contrast and still no oral contrast. Our results indicate that this may be the way to examine IBD patients in the future. 

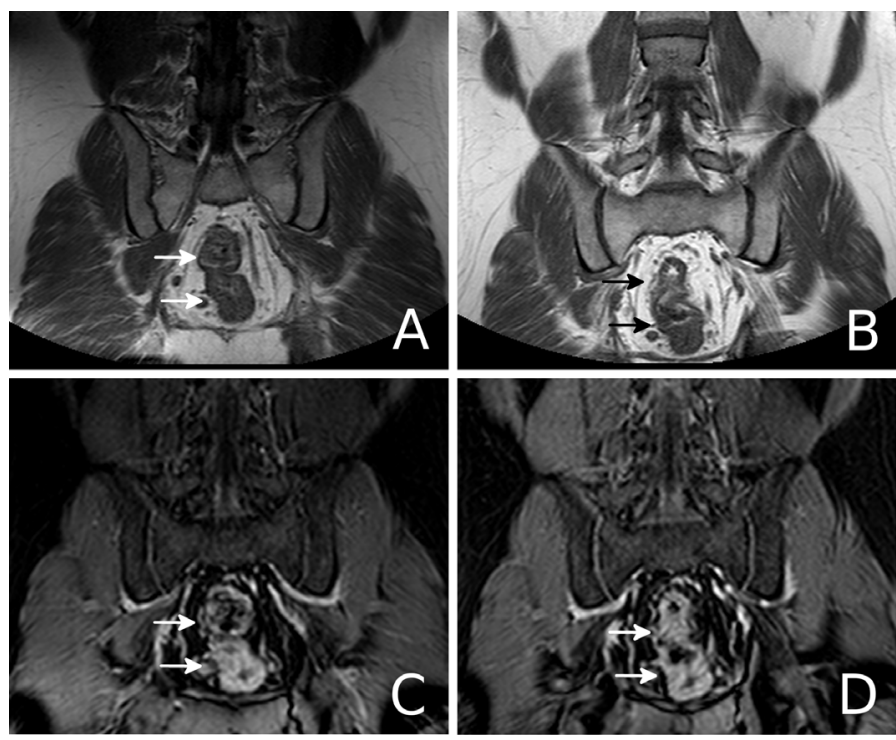

Figure 1. A 42-year-old man recently diagnosed with CD observed blood in every stool. The endoscopy showed active inflammation in sigmoid colon and rectum, verified by biopsy. The FC was $321 \mu \mathrm{g} / \mathrm{g}$. Coronal T2-weighted image showed wall thickening (arrows) of sigmoid colon and rectum in MRI-NOC (A) and MRFT (B). Coronal gadolinium-enhanced image shows mural hyperenhancement and wall thickening at same location, indicating acute inflammation (arrows) in MRI-NOC (C) and MRFT (D).

It will be very convenient for the patients, as they do not have to be at a radiology department one hour prior to the MR examination to drink the oral contrast. The intake of oral contrast is not always easy for this group of patients as they already often have stomach pain.

The kappa value showed good agreement for hyperenhancement in MRFT whereas it was poor to moderate for the other parameters. A low kappa value can be a problem due to the subjective observation of the radiologists. The low number of observers and patients could also explain a low kappa. In diagnostic radiology, multiple observers can be used to avoid low kappa value.

Another convenient test for the patients is FC. It has been suggested as a good marker of mucosal inflammation in IBD patients, which also correlates well with endoscopy findings [8] [9]. Multiple studies found FC to be a better predictor for relapse of IBD rather than degree of bowel inflammation [9]-[11]. Jensen et al. found FC to be equally sensitive in small bowel and colon [12]. They also conclude that $\mathrm{CD}$ can be ruled out in patients suspected of having CD if FC is low. One study states that FC is a better marker of endoscopic activity and postoperative recurrence than clinical activity and the other biomarkers in CD patients [13].

In the current study, the sensitivity for FC was high compared with both MRI protocols. However, it is crucial to remember that the statistics on FC are performed on a per patients basis, whereas in MRI the statistics are performed on a segment-by-segment basis. It is also noteworthy that $\mathrm{p}$-value for $\mathrm{FC}$ is very high $(\mathrm{p}=0.54)$. The specificity and accuracy in both MRI protocols were much better in every aspect compared with FC. This means that both MRI protocols are better than FC at detecting the healthy patients. It is also known that FC is not a specific protein for inflammatory bowel disease as it is also elevated under other conditions [14]. Another issue taken into consideration is the lack of agreement with regard to the cut-off value. El-Etreby et al. had a sensitivity and specificity of $82 \%$ and $85 \%$, respectively in UC patients using a cut-off value of $350 \mu \mathrm{g} / \mathrm{g}$ [15]. Another study used a value of $250 \mu \mathrm{g} / \mathrm{g}$ and concluded that FC showed better correlation with the endoscopic scores in $\mathrm{UC}$ rather than in CD [16]. A study by Jensen et al. used $50 \mu \mathrm{g} / \mathrm{g}$ as the cut-off value in CD and detected a sensitivity of $92 \%$ in the small bowel and $94 \%$ in the colon. They concluded that FC levels were equally sensitive in patients with small bowel or colonic CD [12].

Using the SCCAI for UC patients and HBI for CD patients, it showed that three of the CD patients were clarified as normal as the rest of the patients scored in the mild to moderate range. SCCAI and HBI are based on 
subjective symptoms and therefore reflect the patient's well-being rather than degree of inflammation [17]-[19]. It is widely used on a daily basis in clinics to screen the patients due to further examinations or medication.

Due to the small sample size, the power of the study was limited and no power calculation was performed. Another limitation is the lack of detection of small bowel lesions with either MRI protocol. Furthermore, the lack of oral contrast medium in the colon with MRFT renders the two MRI protocols identical for the colon. To enable a better comparison of MRFT and MRI-NOC, an alteration of this study is needed. Limiting the selection of patients to either CD or UC, thereby restricting the prevalence of lesions to either the small bowel or the colon, enables contrast delivery to be targeted. The small bowel of CD patients would be examined with oral contrast, whereas the colon of UC patients would be examined with contrast delivered by enema. Limitation is also that endoscopy and CE in some cases are done one month before or after the MRI. The disease activity could have changed within this time gap. However, the strength of this study is that we use endoscopy and $\mathrm{CE}$ as the reference standard. We also did the MRFT two days after the MRI-NOC, therefore avoiding the alteration of the lesions between the scans. The FC was also sent to the laboratory one day after the MRI-NOC. The HBI and SCCAI were done on the same day as MRI-NOC.

The location of lesions in the colon combined with the lack of oral contrast in the colon renders MRFT and MRI-NOC functionally identical. However, the idea for this study is unique and needs to be developed further in a large group of patients.

\section{Acknowledgements}

The authors want to express their gratitude to Jakob Moller, M.Sc., for assistance with MRI protocol, Rasmus Hvass Hansen, M.Sc and PhD, for statistics and Krister Reinholdt Nielsen, Cand.scient, for language support. I would like to thank laboratory assistant Vibeke Voxen Hansen for the analysis of faecal calprotectin and CALPRO AS for sponsoring the ELISA kits.

\section{References}

[1] Jakobsen, C., Wewer, V., Urne, F., Andersen, J., Faerk, J., Kramer, I., et al. (2008) Incidence of Ulcerative Colitis and Crohn's Disease in Danish Children: Still Rising or Levelling Out? Journal of Crohn's and Colitis, 2, 152-157. http://dx.doi.org/10.1016/j.crohns.2008.01.006

[2] Shivananda, S., Lennard-Jones, J., Logan, R., Fear, N., Price, A., Carpenter, L., et al. (1996) Incidence of Inflammatory Bowel Disease across Europe: Is There a Difference between North and South? Results of the European Collaborative Study on Inflammatory Bowel Disease (EC-IBD). Gut, 39, 690-697. http://dx.doi.org/10.1136/gut.39.5.690

[3] Pedersen, N., Elkjaer, M., Duricova, D., Burisch, J., Dobrzanski, C., Andersen, N.N., et al. (2012) eHealth: Individualisation of Infliximab Treatment and Disease Course via a Self-Managed Web-Based Solution in Crohn's Disease. Alimentary Pharmacology Therapeutics, 36, 840-849. http://dx.doi.org/10.1111/apt.12043

[4] Riccioni, M.E., Urgesi, R., Cianci, R., Bizzotto, A., Spada, C. and Costamagna, G. (2012) Colon Capsule Endoscopy: Advantages, Limitations and Expectations. Which Novelties? World Journal of Gastrointestinal Endoscopy, 4, 99-107. http://dx.doi.org/10.4253/wige.v4.i4.99

[5] Viera, A.J. and Garrett, J.M. (2005) Understanding Interobserver Agreement: The Kappa Statistic. Family Medicine, 37, 360-363.

[6] R Core Team (2013) R: A Language and Environment for Statistical Computing. R Foundation for Statistical Computing, Vienna. ISBN 3-900051-07-0. http://www.R-project.org/

[7] Jesuratnam-Nielsen, K., Rezanavaz-Gheshlagh, B., Løgager, V.B., Munkholm, P. and Thomsen, H.S. (2015) Plain Magnetic Resonance Imaging as an Alternative in Evaluating Inflammation and Bowel Damage in Inflammatory Bowel Disease-A Prospective Comparison with Conventional Magnetic Resonance Follow-Through. Scandinavian Journal of Gastroenterology, 50, 519-527. http://dx.doi.org/10.3109/00365521.2014.1003398

[8] Røseth, A.G., Aadland, E. and Jahnsen, J.R.N. (1997) Assessment of Disease Activity in Ulcerative Colitis by Faecal Calprotectin, a Novel Granulocyte Marker Protein. Digestion, 58, 176-180. http://dx.doi.org/10.1159/000201441

[9] Costa, F., Mumolo, M.G., Ceccarelli, L., Bellini, M., Romano, M.R., Sterpi, C., et al. (2005) Calprotectin Is a Stronger Predictive Marker of Relapse in Ulcerative Colitis than in Crohn's Disease. Gut, 54, 364-368. http://dx.doi.org/10.1136/gut.2004.043406

[10] Lewis, J.D. (2011) The Utility of Biomarkers in the Diagnosis and Therapy of Inflammatory Bowel Disease. Gastroenterology, 140, 1817-1826. http://dx.doi.org/10.1053/j.gastro.2010.11.058

[11] Benitez, J.M., Meuwis, M.A., Reenaers, C., Van Kemseke, C., Meunier, P. and Louis, E. (2013) Role of Endoscopy, 
Cross-Sectional Imaging and Biomarkers in Crohn's Disease Monitoring. Gut, 62, 1806-1816. http://dx.doi.org/10.1136/gutjnl-2012-303957

[12] Jensen, M.D., Kjeldsen, J. and Nathan, T. (2011) Fecal Calprotectin is Equally Sensitive in Crohn's Disease Affecting the Small Bowel and Colon. Scandinavian Journal of Gastroenterology, 46, 694-700. http://dx.doi.org/10.3109/00365521.2011.560680

[13] Lobatón, T., López-García, A., Rodríguez-Moranta, F., Ruiz, A., Rodríguez, L. and Guardiola, J. (2013) A New Rapid Test for Fecal Calprotectin Predicts Endoscopic Remission and Postoperative Recurrence in Crohn's Disease. Journal of Crohn's and Colitis, 7, e641-e651. http://dx.doi.org/10.1016/j.crohns.2013.05.005

[14] Tibble, J., Teahon, K., Thjodleifsson, B., Roseth, A., Sigthorsson, G., Bridger, S., et al. (2000) A Simple Method for Assessing Intestinal Inflammation in Crohn's Disease. Gut, 47, 506-513. http://dx.doi.org/10.1136/gut.47.4.506

[15] El-Etreby, S., Farag, R., Hakim, H., Ragab, M., Abdeen, H., Alsayed, S., et al. (2013) A New Cutoff Value for Fecal Calprotectin Level in Differentiating Functional from Organic Causes of Chronic Diarrhea. Tanta Medical Journal, 41, 336-341. http://dx.doi.org/10.4103/1110-1415.126199

[16] Nancey, S., Boschetti, G., Moussata, D., Cotte, E., Peyras, J., Cuerq, C., et al. (2013) Neopterin is a Novel Reliable Fecal Marker as Accurate as Calprotectin for Predicting Endoscopic Disease Activity in Patients with Inflammatory Bowel Diseases. Inflammatory Bowel Diseases, 19, 1043-1052. http://dx.doi.org/10.1097/MIB.0b013e3182807577

[17] Tibble, J.A. and Bjarnason, I. (2001) Non-Invasive Investigation of Inflammatory Bowel Disease. World Journal of Gastroenterology, 7, 460-465.

[18] Gaya, D.R., Lyon, T.D.B., Duncan, A., Neilly, J.B., Han, S., Howell, J., et al. (2005) Faecal Calprotectin in the Assessment of Crohn's Disease Activity. QJM, 98, 435-441. http://dx.doi.org/10.1093/qimed/hci069

[19] Maccioni, F., Bruni, A., Viscido, A., Colaiacomo, M.C. and Cocco, A. (2006) MR Imaging in Patients with Crohn Disease : Value of T2- versus T1-Weighted Gadolinium-Enhanced MR Sequences with Use of an Oral Superparamagnetic Contrast Agent. Radiology, 238, 517-530. http://dx.doi.org/10.1148/radiol.2381040244 